\title{
Análise comparativa entre medições in situ e estimativas numéricas na Praia da Cornélia, Costa da Caparica, Portugal *
}

\section{Comparative analysis between in situ measurements and numerical estimations on Cornélia Beach, Costa da Caparica, Portugal}

\author{
M. V. L. Rocha ${ }^{\circledR, 1}$, T. Moura ${ }^{2}$, C. J. E. M. Fortes ${ }^{2}$, R. Capitão ${ }^{2}$, M. M. Bezerra ${ }^{2}$, F. E. Sancho ${ }^{2}$
}

|

\section{RESUMO}

Este artigo aborda a análise comparativa entre as mediçóes de agitação marítima efectuadas na Praia da Cornélia (Costa da Caparica, Portugal), durante os dias 12 a 14 de Maio de 2010, e os resultados numéricos da aplicação do modelo não-linear do tipo Boussinesq, COULWAVE. Esta análise tem como principal objectivo a avaliaçáo do desempenho deste modelo numérico na simulação da propagação de ondas em condiçóes reais, pretendendo evidenciar, deste modo, as suas potencialidades e limitaçóes.

As condiçóes de fronteira do modelo foram geradas com base em séries temporais de elevação medidas a uma profundidade aproximada de $7.6 \mathrm{~m}$ (relativa ao zero hidrográfico - ZH). Os valores numéricos (elevação da superfície livre e a componente transversal da velocidade horizontal) foram obtidos em todo o domínio de cálculo, e em particular, nas posiçóes dos sensores de pressão e de um correntómetro electromagnético colocado junto à linha de costa. Nas posiçôes dos instrumentos são apresentadas análises comparativas no domínio do tempo e estatísticas de conjunto, que servem para a validação das simulaçóes e a quantificação das diferenças observadas entre os resultados numéricos e as mediçóes efectuadas. Analisou-se também a influência nos resultados da escolha de diferentes valores do parâmetro de início da rebentação. Finalmente, apresentam-se também resultados da análise espectral, usando dois métodos espectrais distintos (Fourier e Wavelet), para avaliar a capacidade do modelo COULWAVE na simulação de efeitos não-lineares.

Verificou-se que o modelo simula razoavelmente bem a evolução da onda desde a posição mais ao largo até à praia, antes da ocorrência de rebentaçáo, bem como a velocidade transversal junto à costa. Em geral, tem uma maior capacidade para reproduzir as alturas do que os períodos de onda. É de notar a existência de diferenças por vezes significativas entre os valores medidos e os valores estimados pelo modelo numérico, principalmente nas zonas menos profundas, após a rebentação. No caso da velocidade transversal verifica-se que o modelo simula bastante bem a ordem de magnitude dos valores medidos, mas tem limitaçóes em descrever o seu andamento, verificandose, em geral, a sobrestimação da componente transversal. Ao nível espectral, o modelo é capaz de representar bastante bem a presença das frequências de maior energia registadas, mas tem dificuldades em representar a forma do espectro e a distribuiçáo de energia por períodos ao longo do tempo, principalmente após a rebentaçáo.

Com esta abordagem pretende-se ter uma maior confiança na utilização generalizada do modelo numérico para caracterização da agitação marítima em zonas costeiras e em escalas temporais e espaciais maiores, necessárias numa perspectiva de gestão costeira operacional.

Palavras-chave: Propagação das onda, Rebentação, Modelação Numérica, Praia da Cornélia.

\section{@ - Autor correspondente}

1 - LNEC, Av. do Brasil, 101, 1700-066 Lisboa, Portugal; CESAM, Departamento de Física, Universidade de Aveiro, Campus de Santiago, 3810-193 Aveiro, Portugal e-mail:m.rocha@ua.pt,

2-LNEC, Av.do Brasil, 101,1700-066 Lisboa,Portugal, e-mails: theogrm@gmail.com, jfortes@lnec.pt,rcapitao@lnec.pt, mbezerra@ualg.pt,fsancho@lnec.pt 


\section{ABSTRACT}

This paper presents a comparative analysis between field data collected on Cornélia beach (Costa da Caparica, Portugal), between the $12^{\text {th }}$ and the $15^{\text {th }}$ of May of 2010 and numerical results obtained with the non-linear 2D COULWAVE model. The ultimate aim of this work is to evaluate the capacity and ability of the model to simulate both the measured wave climate conditions and the horizontal velocity.

The COULWAVE numerical model is based on Boussinesq-type equations, obtained from depth-integration of the continuity and momentum equations, assuming a multi-layer concept, and simulates the propagation of strongly non-linear and dispersive waves, across variable-depth zones. Boundary conditions were constrained based on free-surface elevation time series measured at a $7.6 \mathrm{~m}$ depth (Chart datum). Free-surface elevation and cross-shore velocity time series obtained for specific points of the domain were simulated by the model using 30min-long runs, while simulations for the whole domain were only carried on for 300s, due to limited computational capacity.

The numerical results are compared with free-surface elevation time series obtained with pressure sensors and horizontal velocity time series acquired with a current meter positioned near the shoreline. It is also performed a sensibility analysis of the parameter that defines the initial free surface threshold that must be exceeded for a breaking event to initiate: first, it was considered the default value for constant slope beach profiles, 0.65 , and then another value, 0.35 , advocated by previous authors for bar-through beach profiles.

The primary focuses of the data analysis are time-domain analysis and combined statistics (BIAS, RMSE, IC) of significant wave height and period and maximum, minimum, mean and root-mean-square cross-shore horizontal velocities, aiming the validation of the numerical data. It is further presented, for an example-case, the wave energy spectra using two distinct spectral methods (Fourier and Wavelet).

Overall, the model seems to be fairly capable of simulating the wave characteristics across the shoaling zone as well as the cross-shore velocity along the coast. In general, it has a greater capacity to reproduce the heights of the waves than the periods. It should be noted that there are sometimes large differences between the measured values and the values estimated by the numerical model, especially after the wave breaking.

For the cross-shore velocity, the model simulates quite well the right order of magnitude of the measured values, but it has limitations in describing their behaviour, and, in general, there is an overestimation of this cross-sectional component. In the spectral domain, the model is able to represent quite well the presence of the measured higher-energy frequencies recorded, but presents difficulties representing the spectrum shape and the distribution of energy for periods over time, especially after the break.

This approach aims evaluating the performance of the model on simulating the specific conditions of this particular field place, but also, in a more general way, it intends to enhance the confidence on further applications of COULWAVE to wider and longer spatial and temporal scales, which is fundamental for coastal management purposes.

Keywords: Wave propagation, Wave breaking, Nearshore currents, Numerical modeling, Cornélia beach.

\section{INTRODUÇÃO}

A zona costeira é uma região de dinâmica complexa, fruto da interacção de diversos agentes naturais, entre os quais o vento, a maré e as ondas gravíticas, particularmente em praias mesotidais.

As ondas geradas pelo vento são consideradas o agente forçador dominante na hidro e morfodinâmica costeira. Com efeito, na sua propagação em zonas costeiras, as ondas podem sofrer os efeitos de diversos fenómenos físicos complexos (como por exemplo, os associados à variação da profundidade, à difracção, à ocorrência de rebentaçáo, à dissipação de energia por atrito de fundo, à sua reflexão total ou parcial em obstáculos) que assumem uma importância fundamental em toda a morfodinâmica da zona costeira, bem como no comportamento de estruturas marítimas aí implantadas. Com efeito, as alteraçóes da fisiografia costeira, as modificações dos balanços sedimentares, problemas como a erosão costeira e consequente recuo da linha de costa, o assoreamento de estuários, lagunas ou zonas portuárias e a destruição de estruturas marítimas são consequência directa ou indirecta do clima de ondas que se verifica na zona costeira. Como tal, a capacidade de descrever, simular e prever a transformaçáo das ondas, e deste modo, caracterizar o clima de ondas, torna-se essencial para a compreensão dos processos costeiros e para um correcto planeamento e gestáo de intervençóes costeiras, o que contribui, sem dúvida, para uma gestão da zona costeira de forma integrada e sustentável.
Assim, para um maior conhecimento da agitação marítima, a aquisição de dados de campo para monitorização ambiental in situ e o recurso à modelação numérica têm sido amplamente usados. Com efeito, a monitorização in situ é, sem dúvida, a melhor técnica para a caracterização dos parâmetros de ondas numa dada regiáo costeira, pois os dados obtidos são o resultado dos vários fenómenos que afetam a propagação de ondas que atingem a referida zona costeira.

No entanto, este monitoramento de ondas é feito de forma pontual no espaço e no tempo e requer recursos económicos elevados associados ao investimento inicial dos equipamentos a utilizar e ao custos da sua manutenção, pelo que usualmente não é possível para a maioria das zonas de estudo, com base em medições, definir um regime de agitação marítima nem avaliar os efeitos a médio e longo-prazo da agitação marítima na morfologia e dinâmica sedimentar nessa zona.

Assim, o recurso à modelação numérica para caracterizar os principais processos de propagaçáo de ondas em zonas costeiras é muito vantajoso, pois permite a caracterização espacial da zona a estudar e principalmente uma análise a curto, médio e longo prazo, constituindo, por isso, uma importante ferramenta de gestáo costeira.

Neste âmbito, os modelos baseados nas equaçóes estendidas de Boussinesq permitem uma descrição adequada da evoluçáo das ondas marítimas em zonas de profundidade variável, tendo em conta os efeitos da refracção, difracção, rebentação e de efeitos não lineares. Um exemplo deste tipo 
de modelos é o modelo COULWAVE (Lynett \& Liu, 2002), que se baseia nas equaçóes do tipo Boussinesq deduzidas por Wei et al. (1995a, b). Lynett e Liu deduziram as equações a partir da integraçáo em profundidade das equaçóes de continuidade e movimento, utilizando o conceito de camadas múltiplas (multi-layer) em que a coluna de água é dividida em várias camadas, admitindo-se um determinado perfil de velocidades para cada uma. A precisão do modelo assim desenvolvido depende do número de camadas que se considera permitindo a sua utilização em águas profundas.

Apesar das inúmeras vantagens do modelo, e tal como qualquer modelo numérico, este tem algumas limitações decorrentes das suas equaçóes-base e da formulação numérica adoptada. Assim, como em cada camada se admite uma determinada aproximação polinomial e com tal se integra as equaçôes na vertical, náo é possível obter com o modelo a estrutura vertical correcta da velocidade das partículas. Este aspecto é particularmente importante quando se tem interesse no transporte de sedimentos e, consequentemente, na morfodinâmica e dinâmica costeira. Por outro lado, fenómenos como a rebentação são incluídos no modelo através da adição de termos à equaçáo original, que dependem de um conjunto de parâmetros que devem ser calibrados para cada caso de estudo, recorrendo, por exemplo, a dados adquiridos in situ. Torna-se assim importante avaliar quais as implicações que tais limitações podem ter no desempenho do modelo COULWAVE, principalmente quando este é aplicado a situaçóes reais.

É entâo neste âmbito, que se insere o presente trabalho. Assim, a existência de um conjunto muito vasto e variado de mediçóes in situ realizadas na Praia da Cornélia (Costa da Caparica, Portugal) de 12 a 14 de Maio de 2010 no âmbito de um projecto de investigaçáo, o projecto BRISA, e a vontade de avaliar o desempenho deste modelo numérico na caracterização da agitação marítima numa situação real, e, assim, reforçar a confiança na sua utilização generalizada em estudos de morfodinâmica e dinâmica costeira a médio e longo prazo, justificaram o presente trabalho, que consistiu na análise comparativa entre as medições de agitação marítima locais e as estimativas produzidas por esse modelo.
Deste modo, identificando as potencialidades e limitaçóes da utilização do modelo COULWAVE, i.e. o seu desempenho em situaçóes reais, este pode constituir uma ferramenta da gestão costeira integrada.

Neste artigo, descreve-se sucintamente a zona de estudo, a Praia da Cornélia e campanha de recolha de dados realizada. Segue-se a aplicação do modelo COULWAVE, incluindo uma breve descrição das suas características e condiçóes de cálculo. Apresentam-se as análises comparativas entre resultados numéricos e as mediçôes efectuadas. $\mathrm{O}$ artigo termina com as conclusóes.

\section{A PRAIA DA CORNÉLIA, COSTA DA CAPARICA}

A praia da Cornélia situa-se na Costa da Caparica, na costa Oeste de Portugal, Figura 1. Trata-se de uma praia exposta, arenosa, com comportamento morfodinâmico intermédio a dissipativo, com relativa protecção às ondas dominantes de NW, dada a acção, a Norte, do Cabo da Roca. Esta protecçáo confere-lhe um regime de agitação, na rebentação, com energia dominante moderada, podendo ser elevada durante tempestades, sobretudo de Oeste e SW.

Esta praia foi seleccionada para o estudo em causa por ser representativa de praias expostas com comportamento morfodinâmico intermédio em regime mesotidal, o que corresponde largamente às praias dominantes em Portugal e a nível mundial. $O$ modelo ficará assim validado para condiçôes de agitação moderada, em regime de maré e comportamento morfodinâmico que abrangem uma vasta gama de praias a nível mundial.

No âmbito do projecto de investigação BRISA ("BReaking waves and Induced SAnd transport"), foi realizada uma campanha de medições entre os dias 12 e 14 de Maio de 2010 que teve como objectivos a aquisição de dados hidrodinâmicos e morfológicos para: i) testar e validar modelos numéricos e ii) melhorar o conhecimento da dinâmica na rebentação e a sua influência no transporte de sedimentos e na morfodinâmica da praia em estudo. Dispunha-se assim de um vasto e variado conjunto de medições adequado a ser utilizado na validação de modelos numéricos.
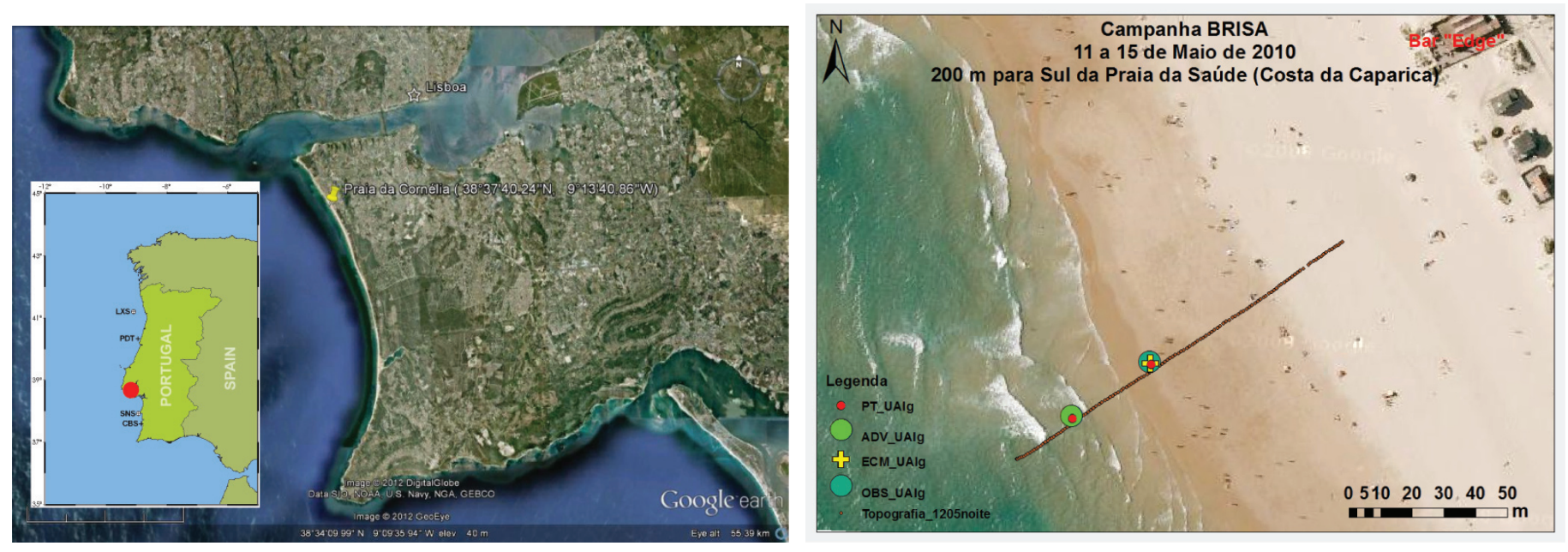

Figura 1. Localização da campanha de campo do Projecto BRISA (Google Earth).

Figure 1. Location of the field campaign of BRISA Project (Google Earth). 
Durante a campanha, foram efectuadas mediçóes da elevação da superfície livre com transdutores de pressão (Pressure Transducers, PT), da velocidade da corrente, com correntómetros (Electromagnetic Current Meters, ECM) e com um medidor acústico de velocidades por efeito de Doppler (Acoustic Doppler Velocimeters, ADV), da turbidez e da concentração de partículas em suspensão, com sensores ópticos (Optical Backscatter Sensors, OBS). Todos os equipamentos foram colocados ao longo de um perfil de praia em estruturas $\mathrm{H}$ identificadas de 1 a 5 , (Figura 2a e 2b) que foram geo-referenciados com um sistema RTK-DGPS sincronizado com o Observatório Astronómico de Lisboa.

A cerca de $-7.6 \mathrm{~m}(\mathrm{ZH})$ de profundidade foi colocada uma poita com um transdutor de pressão, aproximadamente alinhado com o perfil de praia onde foram colocados os equipamentos na face de praia.

No presente trabalho foram apenas analisados os dados de elevação da superfície livre e de velocidades de escoamento referentes às séries temporais de elevação da superfície livre obtidas pelos transdutores de pressão ao largo (PT00) e nas estruturas H1 e H5 (PT01 e PT02) e da velocidade transversal (cross-shore) à costa, obtida com o sensor ECM (estrutura H1), cujo posicionamento é dado na Tabela 1.

\section{APLICAÇÃO DO MODELO COULWAVE}

\subsection{O modelo COULWAVE}

O modelo COULWAVE, Lynett \& Liu (2002), é um modelo 2D de diferenças finitas para a simulação da propagaçáo de ondas fortemente não-lineares (em que a razão entre a amplitude da onda e a profundidade pode ser de ordem até 1) e dispersivas, em zonas de profundidade variável. As equaçóes do modelo, do tipo Boussinesq, são deduzidas a partir da integração em profundidade das equaçóes de continuidade e movimento, utilizando o conceito de camadas múltiplas (multi-layer). Em cada camada admite-se um dado
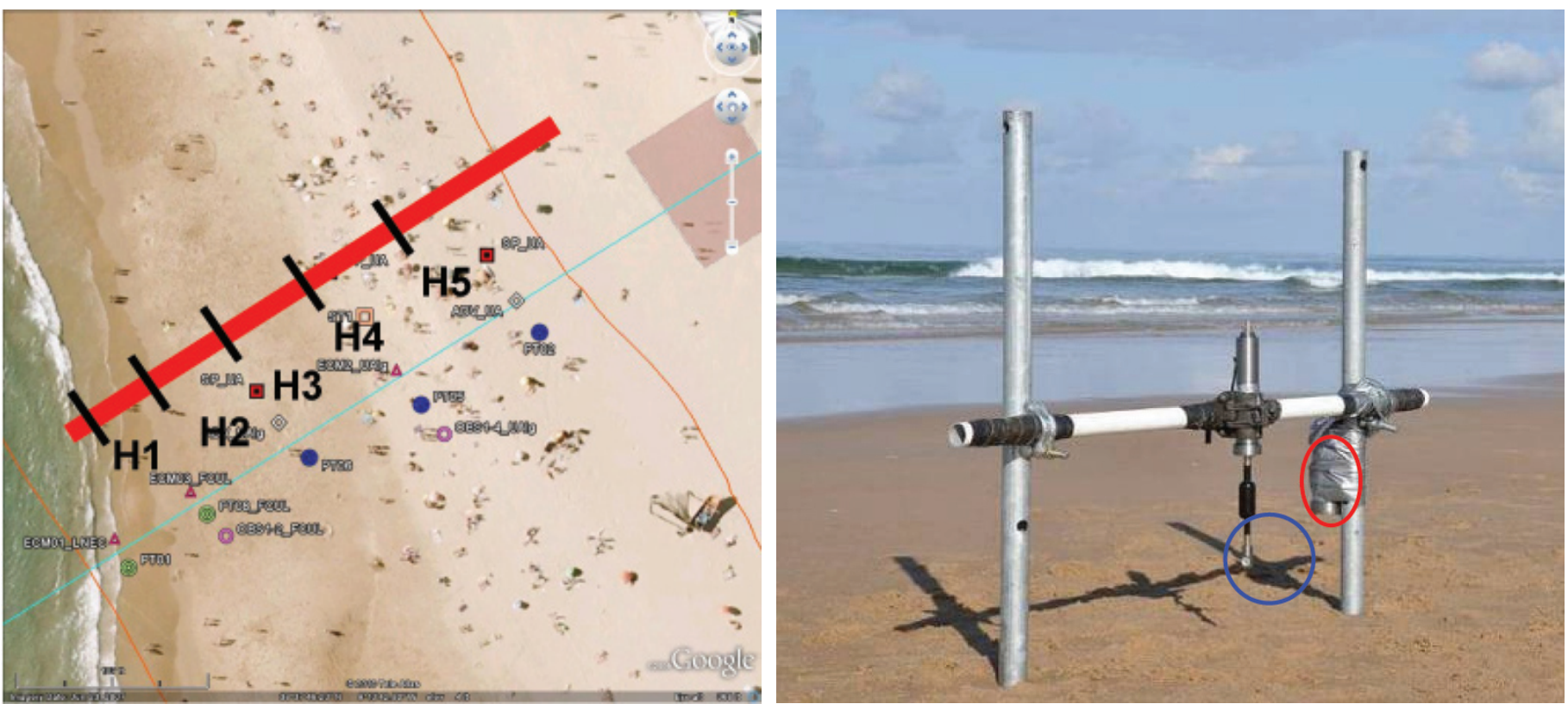

Figura 2. a) Localização do perfil da praia onde foram posicionados os equipamentos (Google Earth) e b) Estrutura H1: Círculo azul: Correntómetro electromagnético (ECM); Círculo vermelho: Transdutor de pressão (PT01).

Figure 2. a) Location of the beach profile where the instruments where positioned (Google Earth). b) H1 structure: Blue circle: Electromagnetic current meter (ECM); Red circle: Pressure transducer (PTO1).

Tabela 1. Identificação, posição dos instrumentos: PT00, PT01, PT02 e ECM.

Table 1. PTOO, PTO1, PTO2 and ECM instruments identification and positioning.

\begin{tabular}{ccccc}
\hline Estrutura & Identificaçáo & X (Easting) & Y (Northing) & $\mathbf{Z}(\mathbf{Z H})$ \\
\hline H1 & PT00 & -95847.090 & -115311.000 & -7.600 \\
\hline H1 & PT01 & -95357.825 & -114985.584 & 1.506 \\
\hline H5 & PCM & -95357.492 & -114985.802 & 1.549 \\
\hline
\end{tabular}


perfil de velocidades, através do uso de funçóes quadráticas com valores iguais na interface que divide a coluna de água. Esta aproximação conduz a um sistema de equaçóes sem as derivadas espaciais de ordem elevada resultantes do uso de funções polinomiais de ordem superior, que é normalmente utilizado na dedução das equações de Boussinesq. Com estes perfis de velocidade, que coincidem na fronteira entre camadas, é deduzido um conjunto de equaçóes que permite estender a aplicabilidade do modelo a águas muito profundas e apresentar características lineares até $\mathrm{kh} \sim 8$ e um comportamento não-linear de $2^{\mathrm{a}}$ ordem até kh -6 (sendo $\mathrm{k}$ o número de onda e $\mathrm{h}$ a profundidade). Contudo, como admite aproximaçóes para a distribuição vertical da velocidade em cada uma das camadas em que é dividida a coluna de água, variações significativas do fundo podem não ser correctamente simuladas pelo modelo.

Seguindo o procedimento de Kennedy et al. (2000), são introduzidos termos adicionais nas equaçóes, de modo a serem tidos em conta o atrito de fundo, a rebentação de ondas e a geração de ondas no interior do domínio. Além disso, são incluídos termos de profundidade dependentes do tempo para serem consideradas variações do perfil de fundo no tempo, devidas à ocorrência de um deslizamento ou de um sismo.

A resolução das equações referidas é semelhante à formulação apresentada por Wei et al. (1995) utilizando um esquema previsor-corrector de Adams-Bashforth. O esquema de diferenças finitas consiste num esquema explícito de Adams-Bashforth de $3^{\mathrm{a}}$ ordem no tempo para o passo previsor e implícito de $4^{a}$ ordem no tempo para o passo corrector. Para as derivadas espaciais, são utilizadas diferenças finitas centrais com uma precisão de $4^{\mathrm{a}}$ ordem. As derivadas espaciais e temporais de ordem superior são calculadas com uma precisão de $2^{\mathrm{a}}$ ordem. $\mathrm{O}$ modelo é formalmente preciso até $\Delta \mathrm{t}^{4}$ no tempo e $\Delta \mathrm{x}^{4}$ no espaço. A diferença relativamente a Wei \& Kirby (1995) refere-se a alguns termos não-lineares dispersivos e à existência de termos adicionais, devidos à dependência temporal da profundidade.

Para as fronteiras exteriores, são aplicados dois tipos de condiçóes: reflexão total e radiaçáo. No primeiro caso utiliza-se a metodologia de Wei \& Kirby (1995), enquanto para a radiação ou condição de fronteira aberta, usa-se um esquema de fronteira absorvente (sponge layer), de acordo com Kennedy et al. (2000).

Os dados de entrada do modelo COULWAVE encontramse descritos em Lynett \& Liu (2002). Os resultados fornecidos pelo modelo consistem em séries temporais e espaciais da elevação da superfície livre e velocidades horizontais nos pontos do domínio definidos pelo utilizador.

\subsection{Condiçóes de aplicaçáo do modelo COULWAVE}

As condiçóes de onda incidentes introduzidas no modelo COULWAVE corresponderam a ondas regulares com as características dos valores registados no sensor PT00 durante os dias de medição, de 12 a 14 de Maio. Nestes cálculos, a direcção da onda foi considerada perpendicular ao domínio computacional, uma vez que, apesar de esta opção constituir uma considerável simplificação, por impossibilidade do equipamento não foram registadas as direcçóes de onda na sua posição.
Simularam-se 127 períodos caracterizados por diferentes condiçôes de agitação marítima, cujas características das ondas ( $\mathrm{Hs}$ e Ts) foram definidas com base na análise temporal dos valores registados pelos instrumentos em cada período de 30 minutos. O nível de maré foi imposto de acordo com a estimativa do nível de maré do Instituto Hidrográfico para o período da campanha.

Em termos de cálculos numéricos, essas características foram simuladas pelo modelo durante 30 minutos para obtenção de resultados numéricos (elevação da superfície livre e velocidade horizontal) em pontos específicos do domínio, nomeadamente na posição dos instrumentos de medição. Em todos os casos, os resultados foram obtidos pelo modelo numérico com um intervalo de tempo de cerca de $0.1 \mathrm{~s}$.

A zona de estudo foi descrita por uma malha regular de espaçamento aproximadamente constante $\Delta x=\Delta y \approx 2.0 \mathrm{~m}$, com dimensão de $900 \mathrm{~m}$ na direcção $\mathrm{OX}$ (perpendicular à costa), e 858 m na direcção OY (paralela à costa) (Figura 3). Esta malha é gerada pelo modelo com base num número mínimo de pontos por comprimento de onda, 30 (nestas simulaçóes), definido consoante o período de onda. A batimetria considerada pela malha correspondeu a uma aproximação da batimetria real.

Foi também adicionada ao domínio, para o largo, uma zona de profundidade constante na qual se efectuou a geração da onda através do método da função-fonte, Wei et al. (1995). Foram usadas nas extremidades laterais (barlamar e sotamar) do domínio de cálculo, condiçôes fronteira de absorção (camadas de $\mathrm{L} / 2=\mathrm{gT}^{2} / 4 \pi$, sendo $\mathrm{L}$ o comprimento de onda e $\mathrm{T}$ o período) com o objectivo de absorver a energia das ondas que atingem estas regióes. Nas restantes fronteiras, por simplicidade, foram consideradas condiçôes de reflexão total.

Em todas as simulaçóes foi considerada apenas uma camada e utilizadas equaçôes completamente não-lineares (com termos dispersivos não-lineares) com atrito de fundo (coeficiente de atrito $=1.0 \times 10^{-2}$ ). $\mathrm{O}$ número de Courant admitido foi de 0.4 e a função-fonte foi colocada na posição $\mathrm{x}=100 \mathrm{~m}$. As 127 simulaçôes foram realizadas assumindo dois valores diferentes para o parâmetro de início da rebentação, $\mathrm{A},\left(\zeta_{t}^{\varphi}==A \sqrt{g H}\right)$, tendo sido, primeiramente, adoptado o valor de $A=0.65$, sugerido no manual do modelo, para praias de perfil com inclinação constante, o qual foi posteriormente alterado para $\mathrm{A}=0.35$, valor sugerido por Chen et al. (2000) para praias de perfil barra-fossa (caso da praia considerada). Para os restantes parâmetros do modelo, consideraram-se os valores sugeridos pelo manual (Lynett \& Liu, 2002).

Os resultados numéricos foram obtidos para as posiçóes correspondentes aos instrumentos PT00 ( $\mathrm{x}=100 \mathrm{~m}, \mathrm{y}=400$ $\mathrm{m}, \mathrm{z}=-7.6 \mathrm{~m})$, PT01 ( $=790 \mathrm{~m}, \mathrm{y}=430 \mathrm{~m}, \mathrm{z}=1.706 \mathrm{~m})$ e PT02 ( $x=868 \mathrm{~m}, \mathrm{y}=400 \mathrm{~m}, \mathrm{z}=3.231 \mathrm{~m})$. As profundidades encontram-se referidas ao Zero Hidrográfico. 
a)

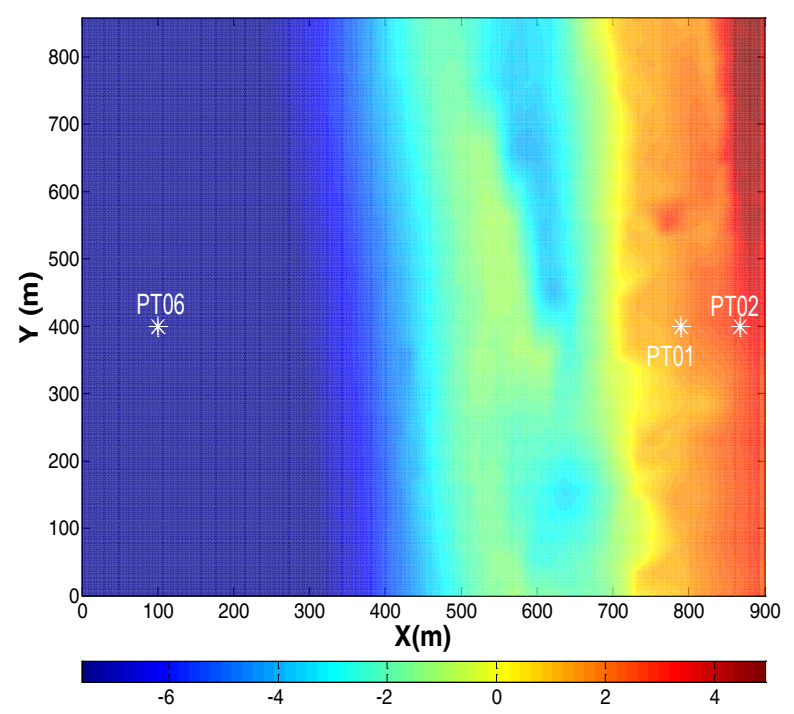

b)

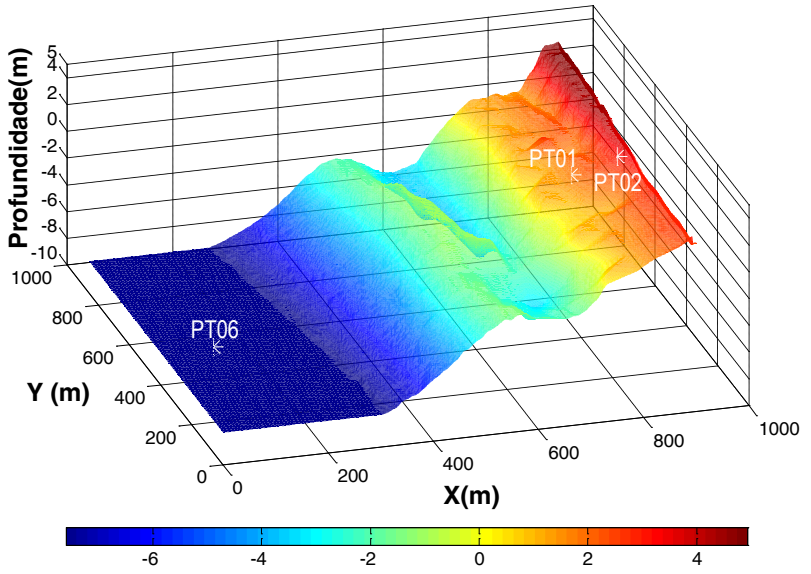

Figura 3. a) Representação em planta do domínio e batimetria reais e b) Representação 3D da batimetria do domínio. Os marcadores brancos assinalam os pontos de cálculo da malha correspondentes aos instrumentos PT00, PT01 e PT02 (respectivamente, do largo para a costa).

Figure 3. a) Representation, from a top-view, of the simulation real domain and bathymetry. b) 3D representation of the domain bathymetry. White markers mark PTOO, PTO1 and PTO2 grid-points location (respectively, from the most offshore location to the beach).

\section{RESULTADOS - ANÁLISE DO DESEMPENHO DO MODELO}

Nas posições PT01 e PT02 foram comparados: (i) os valores numéricos e medidos de alturas e períodos de onda significativos, para o período compreendido entre os dias 12 a 14 de Maio de 2010; (ii) os parâmetros estatísticos, nomeadamente o desvio (BIAS), erro quadrático médio (RMSE) e índice de concordância (IC), para as alturas e os períodos de onda significativos, para o período em estudo e (iii) os espectros de energia obtidos para um único período de 30 minutos, com início às 3:00h do dia 13-05, utilizando os métodos de análise espectral de Fourier e de Wavelet.

Com base nos valores de velocidade horizontal, foram comparados na posição do ECM, os valores numéricos e medidos de: (i) velocidades máximas, mínimas, médias e médias quadráticas no período de 12 a 14 de Maio de 2010, para a componente transversal e (ii) parâmetros estatísticos, novamente o desvio, erro quadrático médio e índice de concordância, para as velocidades máximas, mínimas, médias e médias quadráticas, para o período em estudo. Apenas a componente transversal da velocidade foi considerada, uma vez que a direcção das ondas impostas no modelo é perpendicular à costa, sendo portanto esta a componente da velocidade mais relevante de se analisar.

Nestes cálculos, os primeiros $200 \mathrm{~s}$ de simulação não foram considerados. Além disso, com excepção da análise espectral, em todas as análises são apresentados resultados considerando valores do parâmetro de início da rebentação de $A=0.35$ e $A=0.65$.

\subsection{Elevaçáo da superfície livre}

\subsubsection{Análise temporal e estatística de conjunto}

A Figura 4 apresenta a comparação entre as variaçóes de Hs e Ts ao longo do período registado pelos transdutores, PT01 (Figura 4c e Figura 4d) e PT02 (Figura 4e e Figura 4f) e os resultados simulados pelo modelo, para valores de $A=0.35$ e $A=0.65$. Apresentam-se também nesta figura os valores observados no sensor PT00 (Figura 4a e Figura $4 \mathrm{~b}$ ) e os valores numéricos aí obtidos (com $\mathrm{A}=0.35$, pois sáo idênticos a $A=0.65)$ com os quais foi forçado o modelo numérico.

A Tabela 2 e a Tabela 3 apresentam os valores dos parâmetros estatísticos calculados entre os resultados numéricos e medidos nos dois sensores PT01 e PT02. Os parâmetros considerados foram (i) o desvio (BIAS), (ii) o erro médio quadrático (RMSE) e (iii) o índice de concordância (IC):

$$
\begin{aligned}
& B I A S==Y_{i}-X_{i} \\
& R M S E==\sqrt{\frac{\sum_{1=}^{n}=1\left(Y_{1}-X_{i}\right)^{2}}{n}} \\
& I C==1-\frac{\sum_{i=}^{n}={ }_{i}\left|Y_{i}-X_{i}\right|^{2}}{\sum_{i=}^{n}={ }_{i}\left(\left|Y_{i}-\bar{X}\right|+\left|X_{i}-\bar{X}\right|\right)^{2}}
\end{aligned}
$$

onde $\mathrm{X}_{i}$ são os valores experimentais (referência), $Y_{i}$ são os valores obtidos pelo modelo, $\bar{X}$ é a média do valor experimental e $\mathrm{n}$ é o número de pontos. $\mathrm{O}$ índice de concordância, IC, varia de zero a um, correspondendo o último a uma maior concordância dos valores numéricos com os valores experimentais. 
Nesta análise estatística foram excluídos os períodos de 30 minutos que apresentavam valores irrealistas (excessivamente elevados quando comparados com os valores registados em situação de preia-mar) de $\mathrm{Hs}$ e Ts nos valores medidos e/ou numéricos, incluindo os valores referentes aos períodos de maré baixa.

Numa análise prévia às mediçóes efectuadas, verificase que no início do período analisado as condiçóes de agitação marítima foram pouco energéticas (Figura 4a e 4b), tornando-se um pouco mais severas no dia 14 de Maio de 2010, sendo esta agitação maioritariamente o resultado da actuação do vento local.

Relativamente aos dados numéricos, é possível verificar-se que a escolha do parâmetro A não revela particular influência nos valores de $\mathrm{Hs}$ e Ts simulados pontualmente, para cada instrumento, uma vez que os resultados obtidos para $A=0.65$ e $\mathrm{A}=0.35$ são praticamente coincidentes ao longo do registo. No entanto, houve situaçóes que corresponderam aos períodos compreendidos entre as $12 \mathrm{~h} 30$ e as $18 \mathrm{~h} 00$ e entre as $18 \mathrm{~h} 30$ e as $19 \mathrm{~h} 30$ do dia 14 de Maio em que considerando $\mathrm{A}=0.65$, o modelo foi incapaz de simular os valores medidos em PT02 e PT01, respectivamente. Também para $A=0.35$ o modelo apresentou dificuldades em simular a rebentação, devido à reduzida altura significativa, registada no sensor mais ao largo durante o período da campanha, que foi imposta ao modelo.

Numa primeira análise, para os sensores PT01 e PT02, é possível observar-se que o modelo é capaz de simular o andamento e a ordem de grandeza dos valores medidos, além de representar a descontinuidade dos registos concomitante com os períodos de maré mais baixa, presente tanto nos registos de altura de onda como nos de período, o que justifica de certa forma os valores de IC próximos de 1 , tanto para Hs como para Ts. No entanto, é necessário cuidado na análise destes parâmetros estatísticos, porque para a construção das amostras se optou por excluir os valores irrealistas de Hs e Ts (numéricos e medidos) e todos os valores registados durante os períodos de maré mais baixa. Tal reduz o tamanho da amostra, e "melhora" os valores dos parâmetros, facto que adquire uma relevância particular no caso do PT02, que se encontrou emerso durante uma parte significativa do período total.

Uma análise mais detalhada aos valores obtidos para o PT01 mostra que o andamento dos valores numéricos de Hs é muito semelhante ao das mediçóes, sendo no entanto notória uma tendência geral de sobrestimação das medições efectuadas. Em relação aos valores de Ts, as diferenças são mais acentuadas e o modelo não acompanha tão bem $\mathrm{o}$ andamento dos valores medidos. Além disso, apresenta uma variação de valores de Ts entre períodos consecutivos, particularmente durante os períodos de preia-mar, que não é táo visível nos resultados numéricos. As conclusões anteriores podem, de certo modo, ser confirmadas na análise dos parâmetros BIAS, RMSE e IC. Em relação ao parâmetro BIAS, com valores quase sempre positivos, confirma-se a tendência perceptível de sobrestimação (mais óbvia, no caso de Ts) dos dados medidos, por parte do modelo. Os valores de RMSE e IC correspondentes a Ts apresentam valores menos próximos dos óptimos que os calculados para $\mathrm{Hs}$, o que denota as maiores dificuldades do modelo numérico em simular esta variável.

Para locais mais acima do perfil da praia, como em PT02, onde as ondas já rebentaram, verifica-se que o modelo revela uma maior dificuldade em simular os dados reais, principalmente para o caso Ts. Note-se que as mediçóes efectuadas com este sensor são caracterizadas por grandes períodos de descontinuidade, correspondentes à emersão do instrumento durante os períodos de maré mais baixa. Assim, existem poucos valores para comparação, sendo a análise condicionada por tal. Em geral, em PT02, a tendência é de os valores de $\mathrm{Hs}$ numéricos subestimarem os medidos, e os períodos simulados sobrestimam os medidos. É também evidente a maior dificuldade do modelo em simular os valores de Hs para o último ciclo de maré do período em análise.

Tabela 2. Valores de BIAS, RMSE e IC para a Hs nos sensores PT01 e PT02. Table 2. Significant wave height BIAS, RMSE and IC values obtained for PTO1 and PTO2 sensors.

\begin{tabular}{c|c|c|c|c|c|c}
\hline \multirow{2}{*}{ Sondas } & \multicolumn{2}{|c|}{ BIAS (m) } & \multicolumn{2}{c|}{ RMSE (m) } & \multicolumn{2}{c}{ IC } \\
\cline { 2 - 7 } & $\mathbf{A = 0 . 6 5}$ & $\mathbf{A = 0 . 3 5}$ & $\mathbf{A = 0 . 6 5}$ & $\mathbf{A = 0 . 3 5}$ & $\mathbf{A = 0 . 6 5}$ & $\mathbf{A = 0 . 3 5}$ \\
\hline PT01 & -0.049 & -0.013 & 0.132 & 0.117 & 0.94 & 0.95 \\
\hline PT02 & 0.103 & 0.126 & 0.086 & 0.088 & 0.89 & 0.88 \\
\hline
\end{tabular}

Tabela 3. Valores de BIAS, RMSE e IC para Ts nos sensores PT01 e PT02. Table 3. Significant wave period BIAS, RMSE and IC values obtained for PTO1 and PTO2 sensors.

\begin{tabular}{c|c|c|c|c|c|c}
\hline \multirow{2}{*}{ Sondas } & \multicolumn{2}{|c|}{ BIAS (m) } & \multicolumn{2}{c|}{ RMSE (m) } & \multicolumn{2}{c}{ IC } \\
\cline { 2 - 7 } & $\mathbf{A = 0 . 6 5}$ & $\mathbf{A = 0 . 3 5}$ & $\mathbf{A = 0 . 6 5}$ & $\mathbf{A = 0 . 3 5}$ & $\mathbf{A = 0 . 6 5}$ & $\mathbf{A = 0 . 3 5}$ \\
\hline PT01 & 0.533 & 0.580 & 1.991 & 1.966 & 0.93 & 0.93 \\
\hline PT02 & 12.543 & 11.919 & 3.648 & 3.029 & 0.88 & 0.94 \\
\hline
\end{tabular}


De um modo geral, as diferenças observadas entre valores numéricos e medidos podem estar relacionadas com (i) a direcção das ondas imposta ser normal à batimetria (o que agrava as condiçóes de agitação nos sensores junto à linha de costa), não correspondendo à direcção das ondas que efectivamente chegaram à Praia da Cornélia no período da campanha; (ii) limitaçóes do próprio modelo numérico: é de notar que a aplicação do modelo em inclinaçóes de fundo da ordem de 1/6 se torna muito exigente, pois as equaçóesbase são integradas no pressuposto de que os fundos são de inclinação suave. Além disso, fenómenos como a rebentação são incluídos no modelo através da adição de um termo de viscosidade turbulenta à equaçáo original, que depende de um conjunto de parâmetros relacionados com o início, fim e duração da rebentação. Estes parâmetros têm de ser calibrados para cada caso de estudo e condiçáo de agitaçáo, sendo assim uma limitação do modelo numérico. No presente trabalho os parâmetros de rebentação foram considerados constantes para todas as condições de agitação incidente, que na realidade variam ao longo do período total, e para as quais cada parâmetro deveria ser calibrado individualmente.

\subsubsection{Análise espectral}

A Figura 5 representa a análise espectral efectuada através da aplicação de dois métodos espectrais (Fourier e Wavelet) aos dados medidos e resultados simulados, para o período de 30 min com início às 3:00 do dia 13 de Maio, nas diferentes posiçóes dos instrumentos. Esta figura permite analisar a capacidade do modelo simular as características náo-lineares das ondas, mostrando tanto as frequências de maior energia associada (Fourier) como a distribuição dessa energia pelas frequências ao longo do período analisado (Wavelet).

Os espectros de Fourier revelam que as frequências de maior energia observadas nos dados medidos são também representadas pelo modelo, em consistência com o forçamento deste, efectuado com uma onda monocromática. Mais ainda, em consequência das transferências de energia não-lineares entre ondas de diferentes frequências, para os dois sensores mais costeiros, PT01 (Figura 5a) e PT02 (Figura 5d), o modelo simula convenientemente os dois picos principais de energia presentes. Em PT01, mantém-se a presença do pico de maior energia correspondente ao período de $10 \mathrm{~s}$, e aparece um novo pico, de menor energia e período, correspondente à segunda harmónica da componente principal. A forma do espectro numérico é, em geral, bastante semelhante ao do das mediçóes. No caso do PT02, os espectros são completamente diferentes dos observados nos outros sensores. Existe ainda energia associada ao período de $10 \mathrm{~s}$, mas há claramente uma transferência de energia para períodos maiores, que assinala a ocorrência de fenómenos não-lineares mais significativos. Neste caso, é clara a dificuldade do modelo numérico em simular a forma do espectro observado nestas circunstâncias, o que é resultado de se estar na zona após a rebentação onde os efeitos não lineares são importantes e o modelo apresenta claras limitaçóes nestas condiçóes quer devido à sua formulação base (integração na vertical) quer devido à forma parametrizada como foi incluída a rebentação.

Os espectros de Wavelet permitem uma análise mais minuciosa da distribuição da energia pelas principais frequências, i.e., tornam possível a análise da importância de cada frequência ao longo do período de tempo analisado. Para os sensores PT01 (Figura 5b e Figura 5c) e PT02 (Figura 5e e Figura 5f) o modelo identifica a principal gama de períodos, a rondar os $10 \mathrm{~s}$ e representa relativamente bem a presença de uma gama de frequências mais altas $(0.2 \mathrm{~Hz})$ em PT01 e mais baixas $(0.05 \mathrm{~Hz})$ em PT02, com menor energia associada. No entanto, os espectros de Wavelet evidenciam uma menor banda de frequências para os resultados do modelo que para os dados, o que é o resultado de ter-se forçado o modelo com ondas regulares (para além das limitaçốes inerentes ao modelo atrás referidas). Este comportamento é ainda mais notório no caso do PT02.

\subsection{Componente transversal da velocidade da corrente}

A Figura 6 apresenta uma comparação entre os valores medidos de velocidade máxima, mínima, média e média quadrática, no ponto PT01, pelo correntómetro ECM e os valores simulados pelo modelo para esse mesmo ponto, para a componente transversal da velocidade. Note-se que não faz sentido analisar a componente longitudinal da velocidade horizontal porque é normalmente resultado da obliquidade da onda, e esta obliquidade não foi modelada neste trabalho. Além disso, as fronteiras reflectivas admitidas no modelo vão induzir circulaçôes médias fechadas o que não é realista.

Tal como para as alturas e períodos, também para a velocidade se calcularam os valores de BIAS, RMSE e IC, presentes na Tabela 4.

A partir da observação da Figura 6 e da Tabela 4 e, em termos de comportamento geral, verifica-se uma grande semelhança entre valores numéricos emedidos da componente transversal medida pelo ECM. Para ambos, a componente transversal atinge valores, em módulo, superiores no sentido da propagação das ondas (positivo), sendo tal representativo de uma onda náo linear, de forma tipo cnoidal ou de Stokes de $2^{a}$ ordem, com cristas mais elevadas e curtas e cavas mais achatadas e longas. A velocidade média é maioritariamente da ordem de 2 a $3 \mathrm{~cm} / \mathrm{s}$, negativa, correspondendo a uma fraca corrente de fundo (undertow). Nos períodos imediatamente antes ou após àqueles excluídos dos gráficos (em que os sensores estavam emersos), a velocidade média é próximo do zero ou positivo, isto é, com resultante na direcção da onda, em virtude de durante essa medição o sensor se encontrar relativamente próximo da superfície livre, devido à baixa profundidade da água, e portanto medir não a corrente de fundo, mas sim uma corrente próxima da correspondente à deriva de Stokes.

Embora os resultados numéricos sejam capazes de representar bastante bem a ordem de magnitude dos valores medidos, demonstram uma maior dificuldade em descrever o seu andamento, exibindo os resultados numéricos maiores e mais frequentes oscilaçôes entre intervalos de 30 minutos consecutivos. Verifica-se que para os valores de velocidade, o modelo consegue fornecer melhores estimativas dos valores médios (Um e Urms) do que dos valores extremos. Tal é confirmado pelos valores estatísticos: obtêm-se valores pequenos de BIAS e RMSE e índices de concordância superiores a 0.7 , associados aos valores médios de velocidade média e de velocidade média quadrática, e valores bastante 
a)

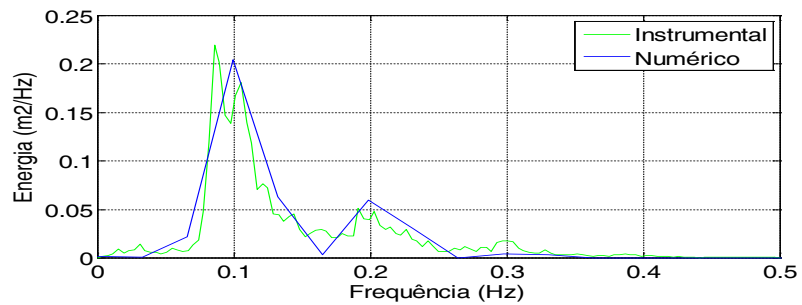

b)
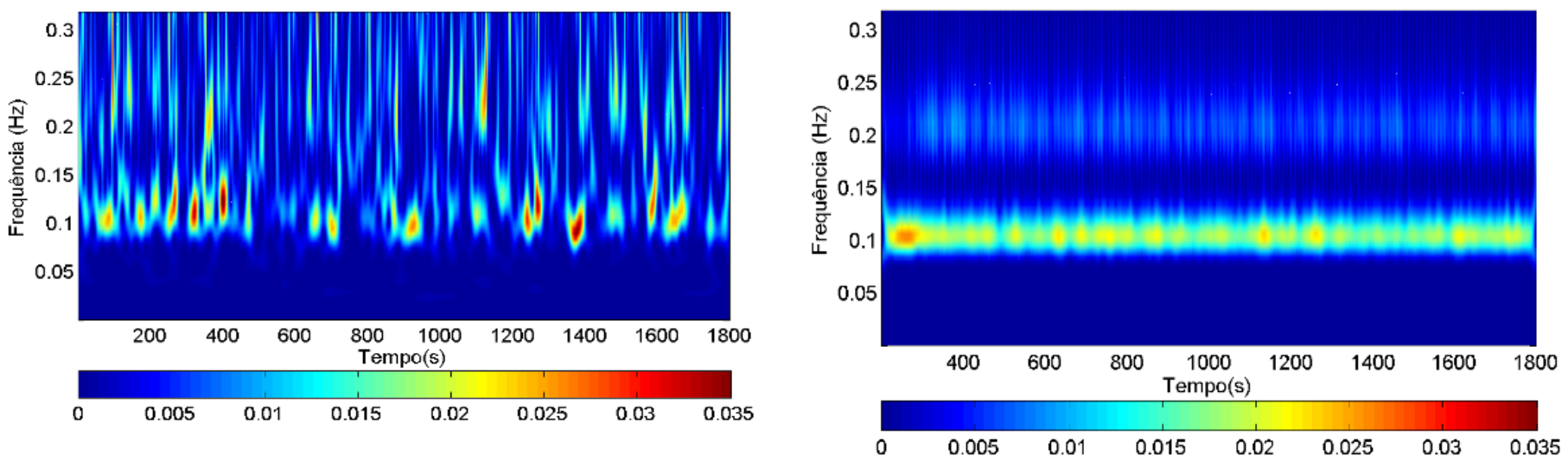

d)

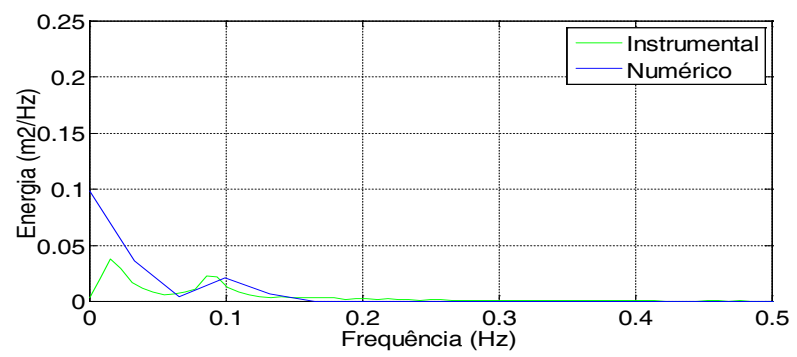

e)

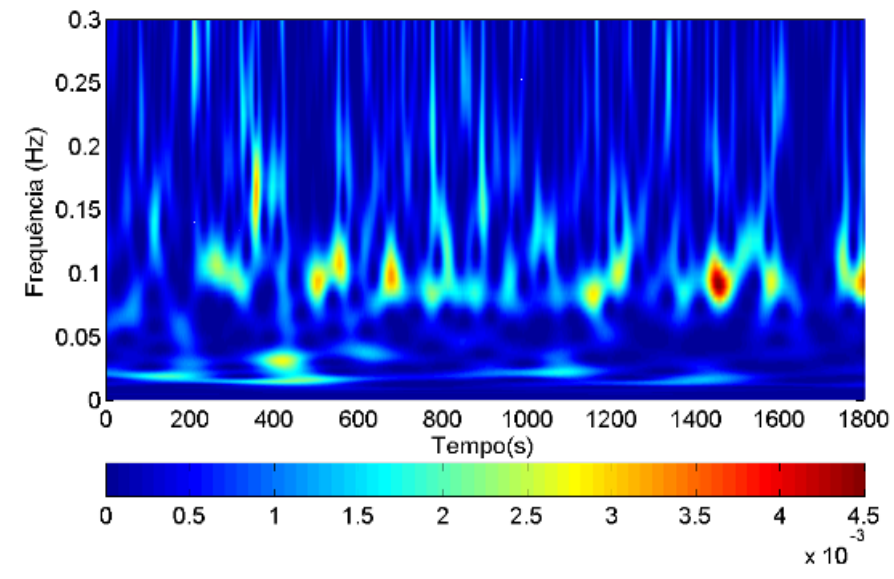

f)

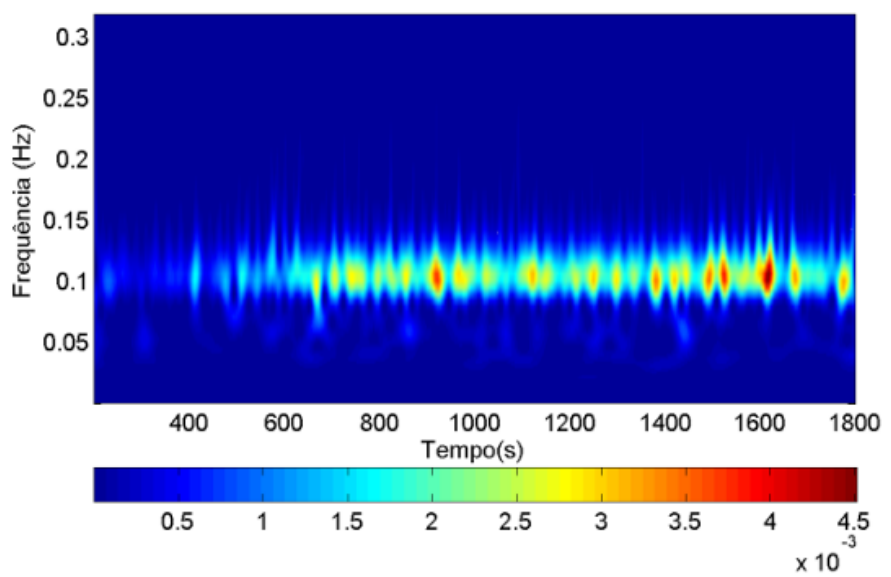

Figura 5. Análise espectral de Fourier (em a e d) dos dados medidos (verde) e resultados simulados (azul), análise espectral de Wavelet (b, c, e, f) dos dados medidos (coluna da esquerda) e resultados simulados (coluna da direita), para o período de 30 min com início às 3:00 do dia 13-05, respectivamente para nos sensores a), b) e c) PT01, d), e) e f) PT02.

Figure 5. Measured and simulated data spectral analysis (Fourier, in a), d) and g) and Wavelet, in the remaining figures), for the 30 minutes period that starts at 3:00, on the 13th of May, respectively for the positions a), b) and c) PTO0, d), e) and f) PTO1 and g), h) and i) PT02. 


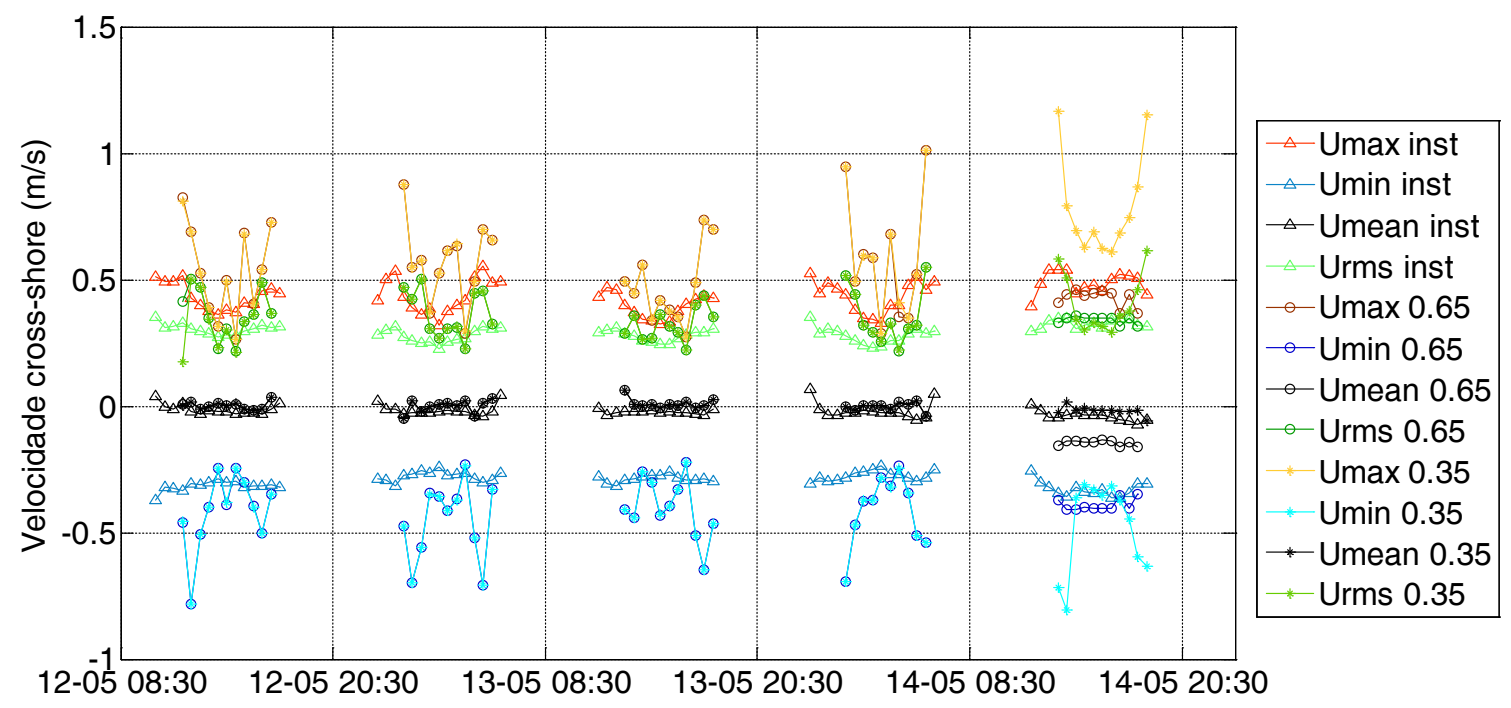

Figura 6. Comparação entre os valores de velocidade máxima, mínima, média e média quadrática, da componente transversal (cross-shore), obtidos com o ECM (marcador: triângulo) e os simulados pelo modelo, para $\mathrm{A}=0.65$ (marcado: círculo) e $\mathrm{A}=0.35$ (marcador: asterisco).

Figure 6. Comparison between measured cross-shore $(U)$ maximum, minimum, mean and root-mean-square velocities, acquired by the ECM (marker: triangle) and the values simulated by the model, considering $A=0.65$ (marker: circle) and $A=0.35$ (marker: star).

Tabela 4. Valores de BIAS, RMSE e IC de Umax, Umin, Umean e Urms, no sensor PT01.

Table 4. BIAS, RMSE and IC of Umax, Umin, Umean and Urms velocities, for PT01 sensor.

\begin{tabular}{|c|c|c|c|c|c|c|}
\hline \multirow{2}{*}{ Sondas } & \multicolumn{2}{|c|}{ BIAS $(\mathbf{m} / \mathbf{s})$} & \multicolumn{2}{c|}{ RMSE $(\mathbf{m} / \mathbf{s})$} & \multicolumn{2}{c|}{ IC (m/s) } \\
\cline { 2 - 7 } & $\mathbf{A = 0 . 6 5}$ & $\mathbf{A = 0 . 3 5}$ & $\mathbf{A = 0 . 6 5}$ & $\mathbf{A = 0 . 3 5}$ & $\mathbf{A = 0 . 6 5}$ & $\mathbf{A = 0 . 3 5}$ \\
\hline Umax & 0.062 & 0.156 & 0.159 & 0.227 & 0.93 & 0.61 \\
\hline Umin & -0.121 & -0.135 & 0.175 & 0.195 & 0.46 & 0.32 \\
\hline Umean & 0.003 & 0.028 & 0.054 & 0.033 & 0.43 & 0.77 \\
\hline Urms & 0.062 & 0.080 & 0.100 & 0.151 & 0.93 & 0.86 \\
\hline
\end{tabular}

elevados de BIAS e RMSE e índices de concordância baixos para os valores extremos dessas variáveis (Umax e Umin).

Verifica-se também, em geral, a sobrestimação dos valores medidos de U, que está provavelmente relacionada com a direcção de propagação das ondas: o modelo foi forçado com ondas perpendiculares à costa, o que resulta em maiores valores de $\mathrm{U}$, contrariamente à direcção real de propagação das ondas na praia, onde a sua obliquidade tenderá a resultar numa componente $\mathrm{U}$ mais fraca.

\section{CONCLUSÓES}

Neste trabalho, descreveu-se e analisou-se a aplicaçáo do modelo COULWAVE às condiçôes de agitação registadas num sensor de pressão posicionado à profundidade de 7.6 m $(\mathrm{ZH})$ durante o período de 12 a 14 de Maio de 2010, na praia da Cornélia (Costa da Caparica). Foram efectuadas comparaçóes entre resultados numéricos e mediçóes efectuadas por sensores de pressão e de velocidade, localizados ao longo do perfil de praia na zona de rebentação.

De um modo geral, concluiu-se que o modelo simula bem a evoluçáo da onda, desde a posição mais ao largo até à praia, antes da ocorrência de rebentação e a velocidade transversal, junto à costa. Após a rebentação, é de notar a existência de diferenças por vezes significativas entre os valores medidos e os valores estimados pelo modelo numérico que estão relacionadas principalmente com as condições de agitação impostas (agitação regular, direcção normal às batimétricas, parâmetros de rebentação constantes ao longo do período de tempo analisado) e com as limitaçóes do próprio modelo (modelo integrado em profundidade). 
Ao nível espectral, o modelo é capaz de representar bastante bem a presença das frequências de maior energia registadas, mas é clara a sua dificuldade em simular a forma do espectro e a distribuição de energia ao longo do período de análise, principalmente após a ocorrência da rebentação onde os efeitos não-lineares são muito importantes.

De um modo geral, a escolha dos dois valores para o parâmetro de início da rebentação, $A=0.65$ e $A=0.35$, não levou a diferenças significativas entre resultados do modelo numérico. No entanto, principalmente para $A=0.65$, houve situações em que o modelo foi incapaz de simular a rebentação e teve dificuldades em simular convenientemente os valores de altura e período significativos da onda e velocidade horizontal correspondentes ao último ciclo de maré do período analisado.

$\mathrm{O}$ trabalho aqui apresentado permitiu obter um modelo de agitaçáo validado e com aplicabilidade elevada, numa vasta gama de praias como as do tipo da praia da Cornélia (praias expostas com comportamento morfodinâmico intermédio em regime mesotidal) que permitirá o seu uso corrente na caracterização da agitação na rebentação, o que se traduz numa mais valia importante para a adequada aplicação de modelos de agitaçáo marítima em gestão costeira.

A imposição de condições de agitação mais próximas da realidade, bem como uma análise mais extensa de sensibilidade aos parâmetros de rebentação intrínsecos do modelo, serão tópicos de trabalho futuro, o que permitirá certamente melhorar os resultados numéricos. Além disso, será também importante a realização de testes de sensibilidade à direcçáo de incidência das ondas impostas, para melhor avaliar a capacidade do modelo em simular as características de agitação e velocidade horizontal resultantes da obliquidade da propagação das ondas para costa. Por outro lado, será também importante avaliar o desempenho do modelo, particularmente a nível espectral, para condições de agitaçáo impostas correspondentes a um espectro irregular de frequências, e não a ondas monocromáticas.

\section{AGRADECIMENTOS}

Este trabalho foi desenvolvido no âmbito do projecto de investigação "BRISA - Interacção entre a rebentação das ondas e o transporte de areias", PTDC/ECM/67411/2006, e dos projectos PTDC/AMB/67450/2006 e PTDC/ ECM/73145/2006, financiados pela FCT - Fundação para a Ciência e a Tecnologia, Portugal.

\section{BIBLIOGRAFIA}

Chen,Q.,Kirby,J.T., Darlymple,R.A.,Kennedy, B.A.\&Chawla, A. (2000) - Boussinesq modeling of wave transformation, breaking, and runup. II: 2D. Journal of Waterways, Port, Coastal and Ocean Engineering, 126(1):48-56. DOI: 10.1061/(ASCE)0733-950X(2000)126:1(48).

Kennedy, B.A., Chen, Q., Kirby, J.T. \& Dalrymple, R.A. (2000) - Boussinesq Modeling of Wave Transformation, Breaking and Runup I: 1D. Journal of of Waterway, Port, Coastal and Ocean Engineering, ASCE, 126(1):39-47. DOI: 10.1061/(ASCE)0733-950X(2000)126:1(39).

Lynett, P. \& Liu P-L.F. (2002) - Modelling wave generation, evolution and interaction with Depth-Integrated, Dispersive Wave equations. COULWAVE Code Manual. 176p., Long Intermediate Wave Modelling Package, Cornell University, Ithaca, NY, U.S.A.. Não publicado.

Wei, G. \& Kirby, J.T. (1995) - A time-dependent numerical codefor extended Boussinesq equations. JournalofWaterway, Port, Coastal and Ocean Engineering, 121(5):251-261. DOI: 10.1061/(ASCE)0733-950X(1995)121:5(251).

Wei, G., Kirby. J. T., Grilli, S.T., Subramanya, R., (1995b). A time-dependent numerical code for extended Boussinesq equations. Journal of Waterway, Port, Coastal and Ocean Engineering, 121(5):251-261. doi: 10.1061/ (ASCE) 0733-950X(1995)121:5(251).

Wei, G., Kirby, J. T., Grilli, S. T., \& Subramanya, R. (1995a) - A fully nonlinear Boussinesq model for surface waves. Part I. Highly nonlinear unsteady waves. Journal of Fluid Mechanics, 294:71-92. DOI: 10.1017/ S0022112095002813. 